\title{
Power Flow Algorithm for Hybrid AC-DC System Equipped With VSC-HVDCs
}

\author{
Sun Tianhe \\ Electronic and Information EngineeringShenyang \\ Aerospace University \\ Shenyang,China \\ e-mail: suntianhe2013@ sina.com
}

\author{
Sun Yanpeng \\ Energy and Environment \\ Shenyang Aerospace University \\ Shenyang, China \\ e-mail: syp_yh@sina.com
}

\author{
Wang Zhujun \\ Electronic and Information Engineering \\ Shenyang Aerospace University \\ Shenyang,China \\ e-mail:1033418669@qq.com \\ Ye Peng \\ Institute of Electric Power \\ Shenyang Institute of Engineering \\ Shenyang,China \\ e-mail: yepeng_126@sina.com
}

\begin{abstract}
The emergence of full-controlled power electronic devices, such as VSC, provides a new direction for the development of HVDC. Based on the analysis of the steadystate model of VSC-HVDC, its mathematical model for Newton method power flow caluclation is developed. This paper proposes a uniform iterative power flow calculation algorithm for systems equipped with VSC-HVDCs. Simulative results for the modified IEEE-14 node test system demonstrate the validity and accuracy of the algorithm.
\end{abstract}

Keywords- VSC-HVDC; Voltage source converter; power electronic ;MTDC; Power flow calculation

\section{INTRODUCTION}

In 1954, the first high-voltage direct current (HVDC) power transmission project was put into industrialized operation. Since the 1990s, with the rapid development of power electronic devices and control technology, voltage source inverter VSC that is on the basis of the whole control type which can be shut off the device type of is more mature. On the basis of this, new type of high voltage direct current transmission (VSC-HVDC) technology has developed. Compared with the traditional transmission which bases on thyristor dc, it has many advantages which can provide to the isolated load power supply directly and is more economy to the load center over a long distance transmission, etc. ${ }^{[1-3]}$ The MTDC which is on the basis of 2 end HVDC can achieve more power and placement by electricity, which has more and more attention.

Currently, as for ac/dc hybrid transmission system of method research of power flow calculation, there are mainly alternative method and unified iterative method. Alternative method can achieve the dc current and ac current iteration separately as well as can inherit the original pure ac power flow calculation program through solving the dc system and ac system respectively. But alternative iteration process did not consider the coupling relationship between dc network and communication network, which is not high accuracy, poor convergence, especially on the multi-terminal dc system and the weak ac system, due to the strong coupling between ac/dc, when people use the alternative method, the deterioration of convergence will be appeared and power flow even are not convergence. ${ }^{[4]}$ On the basis of analysis of VSC - HVDC steady state model, an ac/dc hybrid system power flow alternate algorithm is put forward in references[5]. According to the amount of adjustment system of inverter control, the different combination of $\mathrm{M}$ and phase Angle of the delta, references[6] list four kinds of operational control scheme and for each control scheme that is given, its ac/dc trend of alternative interface equation. Unified iterative method is the trend of dc system equations and equations with tidal current communication system, which can have a unified solution with an unknown quantity in the ac/dc system. This method can calculate the power flow accurately and have a good convergence. According to different control mode VSC, references[7] deduce the corresponding tide correction equation and proposed unified iterative algorithm that is VSC - HVDC ac/dc hybrid system trend and references[8] study the two end of the renewable energy ac-dc power system and power flow calculation. These documents do not use the unified iterative method to analyze multiterminal tide problem of ac/dc hybrid system and the convergence of power flow calculation should be improved.

In this paper, that is based on the principle of VSC HVDC and steady-state model, communication network and $\mathrm{dc}$ network correction equation were deduced respectively. Based on Newton method, it proposed for MTDC unified iterative method of ac/dc power flow calculation method, which achieved with multiterminal dc power flow calculation of power system.follow.

\section{VSC - HVDC STEADY STATE MODEL}

Voltage source inverter connected to the ac bus by converter transformer. For multiterminal of VSC transmission system, the voltage source inverter is connected with the dc circuit. For the ith a VSC, the steady-state model is shown in Fig. 1 . 


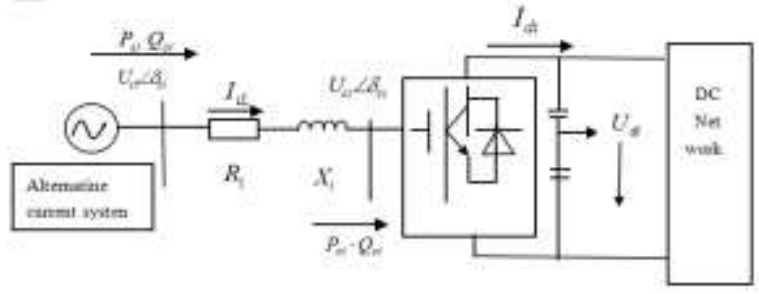

Figure 1. The steady state model of transmission system

In picture 1 , the $\mathrm{i}$ represents the ith an inverter $\mathrm{dc}$ network. Converter internal loss and loss of converter, transformer for $\mathrm{R}$ and the converter transformer impedance for $\mathrm{X}$. The current through the converter transformer is $Q_{s}$ Communication system into the converter transformers active power and reactive power respectively, $P_{s}$ and $Q_{s}$. VSC has absorption of active power and reactive power of $\mathrm{P}_{\mathrm{c}}$ and $Q_{c}$ respectively. Dc voltage for $U_{d}$, dc current for $I_{d}$, ac busbar voltage fundamental component for $U_{t}$, phase Angle for the delta $\mathrm{s}$, inverter ac bus voltage fundamental component for $U_{c}$, phase Angle for the delta c.

When inverter resistance and harmonic component are ignored, it can get VSC and communication system between the transmission of active power and reactive power respectively.

$$
\begin{aligned}
& p_{s}=\frac{U_{t} U_{c}}{X_{i}} \sin \delta_{i} \\
& Q_{s}=\frac{U_{t}}{X_{i}}\left(U_{t}-U_{c} \cos \delta_{i}\right) \\
& \delta_{i}=\delta_{t i}-\delta_{c i}
\end{aligned}
$$

By type (1), (2), active power transmission depends mainly on the delta and reactive power transmission mainly depends on $U_{c}$. By controlling the phase Angle of the delta and $U_{c}$, it can be realized to VSC size and direction of active power as well as reactive power control. While $U_{c}$ by dc voltage $U_{d}$ is associated with mode modulation $\mathrm{M}(0 \leqslant \mathrm{M} \leqslant 1)$ of pulse width modulation (PWM) and Dc voltage utilization and mu $(0<\mu \leq 1)$, which are decided both.

$$
U_{c}=\frac{\mu M}{\sqrt{2}} U_{d}
$$

\section{VSC - HVDC CONVERTER OPERATION CONTROL MODE}

VSC - HVDC uses control switch device, so each inverter has two control volume, PWM modulation $\mathrm{M}$ and phase Angle of the delta. Inverter can be independent regulation of active power and reactive power. To ensure the normal and stable operation of VSC - HVDC, it must make dc network active power balance. The input dc network active power must be equal with the dc network output active power and dc network active power loss. Otherwise, it will cause the system dc voltage fluctuations. Usually, in the multi-terminal dc system, at it must select no more than one inverter control of the dc side voltage, as the balance of active power inverter dc network. So the operation of the VSC - HVDC voltage source inverter control method has four kinds:

a. $U_{d}$ constant dc voltage, ac reactive power $Q_{s}$

b. $U_{d}$ constant dc voltage, ac busbar voltage $U_{s}$. c. Constant communication active power $P_{s}$, ac reactive power $Q_{s}$

d. Ac active power $P_{s}$, ac busbar voltage $U_{s}$.

\section{VSC - HVDC AC-DC POWER FLOW CALCULATION}

The ac/dc transmission system is divided into communication subsystem and dc subsystem analyzed. As for communication subsystem, it can be divided into two classes through the node connected to the inverter: one kind can not directly connect with inverter of ordinary nodes with using subscript a; and the other kind is special nodes and inverter direct connection. With the subscript $t$. the ordinary nodes in the communication system, the node power deviation equation is identical to that of pure ac system.

$$
\begin{aligned}
& \Delta P_{a i}=P_{s a i}-U_{a i} \sum^{j \in i} U_{j}\left(G_{i j} \cos \theta_{i j}+B_{i j} \sin \theta_{i j}\right) \\
& \Delta Q_{a i}=Q_{s a i}-U_{a i} \sum^{j \in i} U_{j}\left(G_{i j} \sin \theta_{i j}-B_{i j} \cos \theta_{i j}\right)
\end{aligned}
$$

As for special node, its power deviation equations include communication system into the ith a inverter power, so

$$
\begin{aligned}
& \Delta P_{t i}=P_{s t i}-U_{t i} \sum^{j \in i} U_{j}\left(G_{i j} \cos \theta_{i j}+B_{i j} \sin \theta_{i j}\right)-P_{s i} \\
& \Delta Q_{t i}=Q_{s t i}-U_{t i} \sum^{j \in i} U_{j}\left(G_{i j} \sin \theta_{i j}-B_{i j} \cos \theta_{i j}\right)-Q_{s i}
\end{aligned}
$$

At this point, the VSC transmission between its ac busbar of active power and reactive power can be written

$$
\begin{aligned}
& P_{s i}=\frac{\mu_{i}}{\sqrt{2}} M_{i} U_{t i} U_{d i} Y_{i} \sin \left(\delta_{i}-\alpha_{i}\right)+U_{t i}^{2} Y_{i} \sin \alpha_{i} \\
& Q_{s i}=\frac{\mu_{i}}{\sqrt{2}} M_{i} U_{t i} U_{d i} Y_{i} \cos \left(\delta_{i}-\alpha_{i}\right)+U_{t i}^{2} Y_{i} \cos \alpha_{i} \\
& Y_{i}=1 / \sqrt{R_{i}^{2}+X_{i}^{2}}, \alpha_{i}=\arctan \left(R_{i} / X_{i}\right)
\end{aligned}
$$

Based on the established steady-state model of VSC HVDC and dc network equation, it can deduce the power flow calculation of dc system equations.

$$
\left\{\begin{array}{l}
\Delta d_{i 1}=P_{s i}-\frac{\mu_{i}}{\sqrt{2}} M_{i} U_{s i} U_{d i}\left|Y_{i}\right| \cos \left(\delta_{i}+\alpha_{i}\right)-U_{s i}^{2}\left|Y_{i}\right| \cos \alpha_{i} \\
\Delta d_{i 2}=Q_{s i}+\frac{\mu_{i}}{\sqrt{2}} M_{i} U_{s i} U_{d i}\left|Y_{i}\right| \sin \left(\delta_{i}+\alpha_{i}\right)-U_{s i}^{2}\left|Y_{i}\right| \sin \alpha_{i} \\
\Delta d_{i 3}=U_{d i} I_{d i}-\frac{\mu_{i}}{\sqrt{2}} M_{i} U_{s i} U_{d i}\left|Y_{i}\right| \cos \left(\delta_{i}-\alpha_{i}\right)+\frac{1}{2}\left(\mu_{i} M_{i} U_{d i}\right)^{2}\left|Y_{i}\right| \cos \alpha_{i} \\
\Delta d_{i 4}=I_{d i}-\sum_{j=1}^{n_{t}} g_{d i j} U_{d i}
\end{array}\right.
$$

In this type: $g_{\text {dij }}$ is the dc network after elimination contact node admittance matrix elements.

Type 5 to 8 with the type 11 , according to the Taylor series expansion, after slightly higher order term, the Newton's method for power flow correction equation is

$\Delta f=-J \Delta x$

As written in matrix form: 


$$
\left[\begin{array}{c}
\Delta P_{a} \\
\Delta P_{t} \\
\Delta Q_{a} \\
\Delta Q_{t} \\
\Delta D_{d}
\end{array}\right]=\left[\begin{array}{ccccc}
N_{a a} & H_{a a} & N_{a a} & H_{a t} & 0 \\
N_{t a} & H_{t a} & N_{t t} & H_{t t} & \frac{\partial \Delta P_{t}}{\partial \Delta x_{d}} \\
L_{a a} & J_{a a} & L_{a t} & J_{a t} & 0 \\
L_{t a} & J_{t a} & L_{t t} & J_{t t} & \frac{\partial \Delta Q_{t}}{\partial \Delta x_{d}} \\
\frac{\partial \Delta D_{d}}{\partial \Delta U_{a}} & 0 & \frac{\partial \Delta D_{d}}{\partial \Delta U_{t}} & 0 & \frac{\partial \Delta D_{d}}{\partial \Delta x_{d}}
\end{array}\right]\left[\begin{array}{l}
\Delta U_{a} \\
\Delta \theta_{a} \\
\Delta U_{t} \\
\Delta \theta_{t} \\
\Delta x_{d}
\end{array}\right]
$$

In the type:N,H,L,J as the Jacobi matrix of pure ac system, $\Delta D_{d}=\left(\Delta d_{i l}, \Delta d_{i 2}, \Delta d_{i 3}, \Delta d_{i 4}\right)^{T}, \Delta x_{d}=\left(\Delta U_{d}, \Delta I_{d}, \Delta \delta\right.$, $\left.\Delta M, \Delta P_{s}, \Delta Q_{s}\right)^{T}$.There is no direct connection with $\mathrm{dc}$ network due to the common node, so $\Delta P_{a}, \Delta Q_{a}$ for $\Delta x_{d}$ partial derivatives of 0 . Similarly, $\Delta D_{d}$ on all nodes of the phase Angle $\theta_{i}$ beg partial derivatives are zero.

For systems with $n$ nodes, if you have $n_{t}$ a voltage source inverter, the type (12) is with a total of $2(n-1)+4 n_{t}$ equation, $2(n-1)+6 n_{t}$ variables. According to the given inverter control mode, it can eliminate $4 n_{t}$ variables, so the type (12) can be solved. Compared with traditional power flow calculation, the system with VSC, Jacobian matrix augmented for $6 n_{t}^{-1}$ order.

Unified iterative method VSC - HVDC ac/dc power flow calculation flow chart is shown in Fig.2.

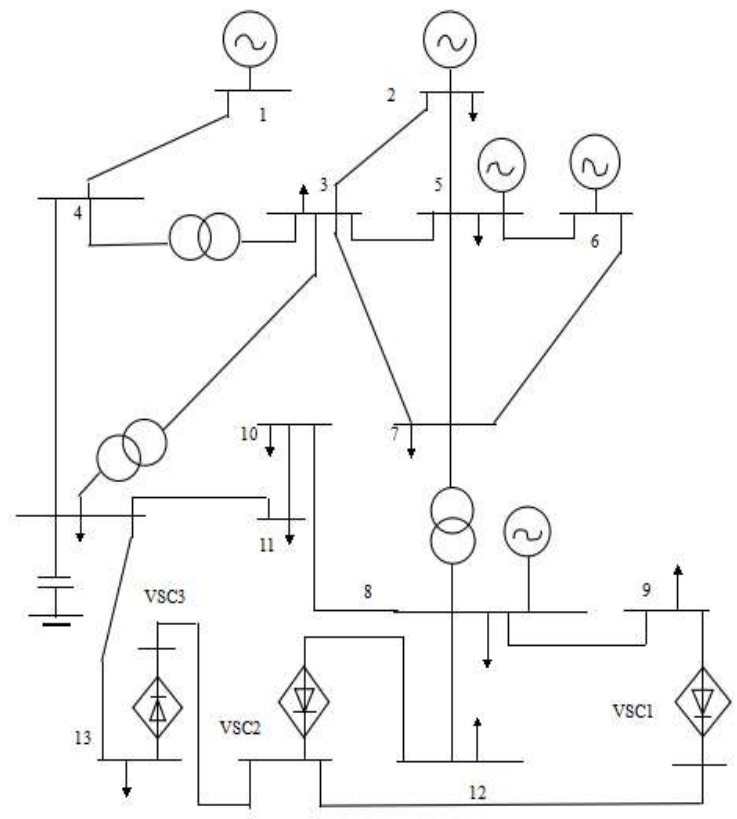

Figure 2. The modified IEEE - 14 nodes ac/dc hybrid system

\section{THE EXAMPLE ANALYSIS}

In this paper, we use the modified IEEE - 14 nodes system as an example to verify the effectiveness of the algorithm. In the Fig .2:

VSC1, VSC2 and VSC3 are on busbar 12, busbar 13 and busbar 14 respectively, $\mathrm{R}=0.006, \mathrm{X}=0.15$. VSC1 adopts constant dc voltage (Ud1ref $=2.0)$,Ac reactive power (Qs1ref $=0.007$ ). VSC2 adopts fixed exchange active power (Ps2ref $=0.0429$ ), ac reactive power control (Qs2ref $=0.0078)$. VSC3 adopts fixed exchange active power $($ Ps3ref $=-0.0552)$, ac reactive power (Qs3ref $=-$ $0.0137)$. Dc resistance network $\mathrm{Rd}=0.03$, dc voltage utilization $\mu=1$.

Power flow calculation results are shown in table 1 and table 2. From the table 2, in this article, the algorithm can converge to the VSC control target. Besides, VSC1, and VSC2 absorbs the active power from the ac power grid and the VSC3 injected active power to the grid for achieving the active power balance in the system.

Program convergences after 4 iterations. References[9] takes the same IEEE - 14 node example in this paper and a flow algorithm with alternating iterative method is presented. Under the condition of the same convergence precision, the number of iterations is 8 times and 4 times respectively, which proves that this algorithm has good convergence.

TABLE I. CALCULATION RESULTS COMMUNICATION TREND

\begin{tabular}{|c|c|c|}
\hline node & voltage amplitude & phase angle / $\left({ }^{\circ}\right)$ \\
\hline 1 & 1.0600 & 0 \\
\hline 2 & 1.0450 & -4.9820 \\
\hline 3 & 1.0100 & -12.7192 \\
\hline 4 & 1.0182 & -10.3142 \\
\hline 5 & 1.0200 & -8.7803 \\
\hline 6 & 1.0700 & -14.2539 \\
\hline 7 & 1.0605 & -13.3424 \\
\hline 8 & 1.0900 & -13.3424 \\
\hline 9 & 1.0535 & -14.9161 \\
\hline 10 & 1.0490 & -15.0842 \\
\hline 11 & 1.0559 & -14.7988 \\
\hline 12 & 1.0554 & -15.1086 \\
\hline 13 & 1.0505 & -15.2135 \\
\hline 14 & 1.0327 & -15.9585 \\
\hline
\end{tabular}

TABLE II. DC CURRENT CALCULATION RESULTS

\begin{tabular}{|c|c|c|c|c|c|c|}
\hline inverter & $\mathbf{U}_{\mathbf{d}}$ & $\mathbf{I}_{\mathbf{d}}$ & $\boldsymbol{\delta} /\left(^{\circ}\right)$ & $\mathbf{M}$ & $\mathbf{P}_{\mathbf{s}}$ & $\mathbf{Q}_{\mathbf{s}}$ \\
\hline VSC1 & 2.000 & 0.0079 & 0.1207 & 0.8608 & 0.0139 & 0.0070 \\
\hline VSC2 & 1.9998 & 0.0214 & 0.3321 & 0.8567 & 0.0429 & 0.0078 \\
\hline VSC3 & 1.9989 & -0.0294 & -0.4678 & 0.8456 & -0.0552 & -0.0137 \\
\hline
\end{tabular}




\section{CONCLUSION}

Ac/dc hybrid transmission system with the VSC flow calculation is the basis of studying the characteristics of VSC - HVDC steady state. It also analyzes the steady state control mode and the corresponding control necessary for protection technology. Due to multiterminal HVDC system's many advantages, the analysis has a realistic significance. ${ }^{[12]}$ This paper presents a ac/dc system power flow calculation and unified iterative method to modify the IEEE system - 14 nodes to calculate, which verifies the accuracy of trend correctness and validity of iterative algorithm in this paper.

\section{ACKNOWLEDGMENT}

I would like to express appreciation for tutor's guidance during the process of selecting and researching subjects, which can pick up more about the content and acquire professional knowledge as well as can improve my abilities of analyzing and innovating. At last, I want to thank for my tutors and classmates who help me complete the paper.

\section{REFERENCES}

[1] Wang Feng-chuan. "HVDC LGHT-DC TRANSMISSION BASED ON VOLTAGE SOURCED CONVERTERS,'Power System Technology, Vol.23,No.4,Apr. 1999.

[2] WEN Jun,ZHANG Yi-gong,HAN Min-xiao,XIAO Xiang-ning. "HVDC BASED ON VOLTAGE SOURCE CONVERTER —A
NEW GENERATION OF HVDC TECHNIQUE." Power System Technology, Vol.27,No.1 ,Jan.2003.

[3] TANG Guang-fu, LUO Xiang, WEI Xiao-guang. "Multi-terminal HVDC and DC-grid Technology," PROCEEDINGS OF THE CSEE,Vol.33 ,No.10 ,Apr.5, 2013.

[4] GE Wei-chun,GU Hong-qun,HE Zhi-yuan. "Overview on Dalian Flexible HVDC Transmission Demonstration Project."Northeast Electric Power Technology, Vol.32, No.2 2012.

[5] J.Reeve and J.Carr,'Review of techniques for multi-terminal HVDC systems."in Proc.Int.Conf. High Voltage DC AC Power Transmission,1973,pp.269-73.

[6] J.Reeve,"Multi-terminal HVDC power-systems,"IEEE Trans. Power App.Syst,Vol.99,No.2,pp729-737,Mar/Apr.1980.

[7] ZHENG Chao,ZHOU Xiao-xin,LI Ruo-mei SHENG Can-hui, "STUDY ON THE STEADY CHARACTERISTIC AND ALGORITHM OFOPOWER FLOW FOR VSC-HVDC.' PROCEEDINGS OF THE CSEE,Vol.25 ,No.6, Mar.2005.

[8] CHEN Qian,YANG Guo-qing,WANG Xun, "AC-DC power flow algorithm for multi-terminal VSC-HVDC systems.” Electric Power Automation Equipment,Vol.25,No.6,Jun.2005.

[9] ZHANG Chao,SHENG Can-hui. "Uniform iterative power flow algorithm forsystems equipped with VSC-HVDC." China Electric Power News, Vol.40,No.7,Jul.2007.

[10] FU Yu,YANG Jian-hua,ZHANG Qi. "Power flow algorithm for AC/DC system with DC sources and loads." Electric Power Automation Equipment, Vol.33,No.1,Jan.2013.

[11] LI Hua. "Research on the Application of Flexible HVDC Power Transmission to Urban Power Network." Northeast Electric Power Technology,Vol.33,No.2,2012.

[12] Christian M.Franck. "HVDC Circuit Breakers:A Review Identifying Future Research Needs."IEEE Transactions On Power Delivery, Vol.26.No.2,April.2011. 